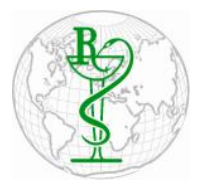

INDO GLOBAL JOURNAL OF

PHARMACEUTICAL SCIENCES

ISSN 2249- 1023

\title{
An Innovative Advancement for Targeted Drug Delivery: Nanosponges
}

\author{
Jyoti P *, Tulsi B, Popin K, Chetna B \\ Department of Pharmaceutics, GISIPS, D. Dun, Uttrakhand Technical University, India
}

\begin{abstract}
Address for
Correspondance

Jyoti P,

jpandey1112@g mail.com

Keywords

Nanosponges;

Targeted Drug

Delivery;

Controlled Drug

Delivery.

ABSTRACT: In general, targeted and controlled drug delivery of drug to specific sites is the significant problem which is being faced by medical practitioner during the drug administration within the body. So, to resolve this problem an innovative drug delivery system was created known as "nanosponges". Nanosponge is the part of advance drug delivery system. It is a specific aiding system for targeted drug delivery of both kind of drugs either it is lipophilic or hydrophilic in a controlled manner. In this review article concluded general introduction of nanosponges, structure, category of nanosponges, significance, preparation methodology and evaluation parameters. Nanosponge is an effective drug delivery system for targeted delivery of variety of drugs and it also enhances solubility of poor water soluble drugs. () 2015 iGlobal Research and Publishing Foundation. All rights reserved.
\end{abstract}

\section{INTRODUCTION}

Targeted drug delivery is a leading technique useful for improvement and advancement in drug dosing, therapeutic efficacy, maximizing therapeutic index of the drug and minimizing side effects. Targeted drug delivery is a part of nanotechnology.

Nanotechnology is defined as creation and manipulation. Such technology resulted in variant of formulations like nanoparticles, nanocapsules, nanospheres, nanosuspensions, nanocrystals, nano-erythosomes etc.

Nanoparticles are available in various forms having at least one dimension in the $1-100 \mathrm{~nm}$ range, like polymeric nanoparticles, solid-lipid nanoparticles, nanoemulsions, nanosponges, carbon nanotubes, micellar systems etc.

Nanosponges are a novel class of nanoparticles with nanostructured hyper branched polymers and few nanometers wide cavities, in which a large variety of substances can be encapsulated. Effective targeted drug delivery has long been a problem for medical researchers i.e., how to get them to the right place in the body and how to control the release of the drug to prevent overdoses. Nanosponges are one of the effective drug delivery system which conquers this problem. The nanosponge drug delivery platform is a network of specific polymers that slowly degrades and thus releases the chosen drug.

Nanosponges are in nano size with sponge like morphology. These are tiny mesh like structures in which large variety of substance can be encapsulated. They have a proven spherical colloidal nature, reported to have a very high solubilization capacity.

Nanosponges can solubilize poorly water soluble drug and provide prolonged release as well as improving drugs bioavailability. Nanosponges are able to load both hydrophilic and hydrophobic drug molecules because of their inner 
Indo Global Journal of Pharmaceutical Sciences, 2016; 6(2): 59-64

hydrophobic cavities and external hydrophilic branching, thereby offering unparalleled flexibility.

Basically, Nanosponges are solid in nature and can be formulated as Oral, Parenteral, Topical or Inhalation dosage forms. For the oral administration, the complexes may be dispersed in a matrix of excipients, diluents, lubricants and anticaking agents suitable for the preparation of capsules or tablets. For topical administration they can be effectively incorporated into topical hydrogel.

\section{STRUCTURE OF NANOSPONGES}

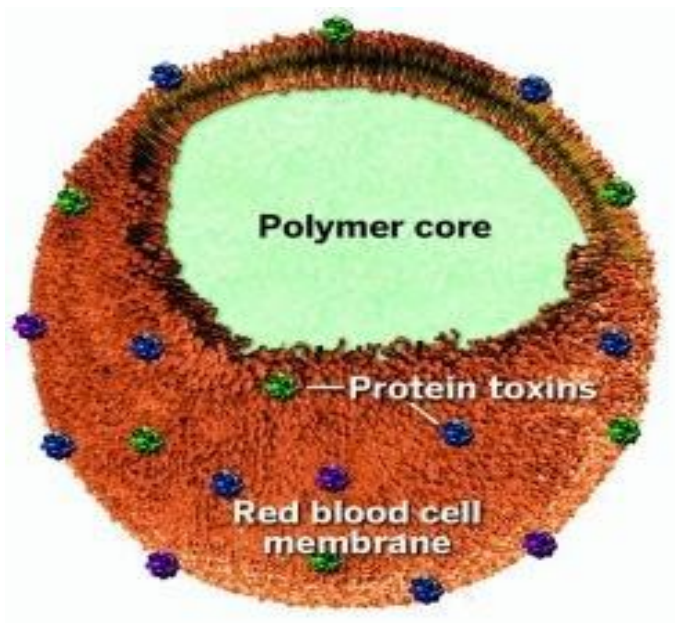

Fig 1: Polymer based Nanosponge.

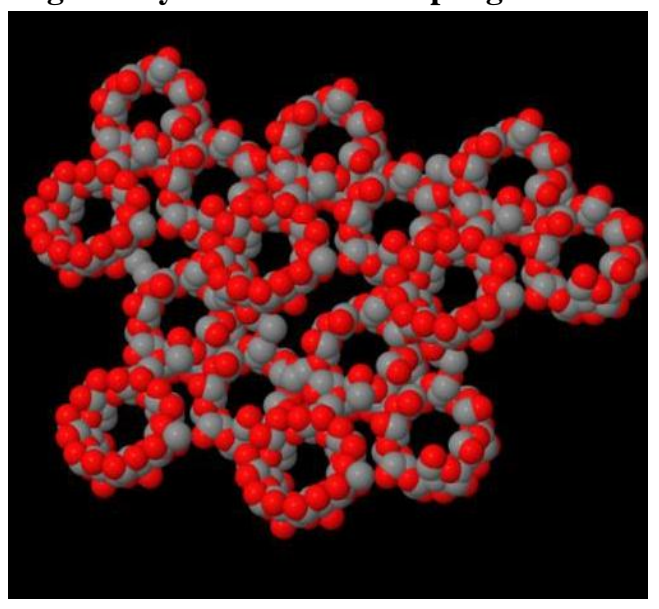

Fig 2: Cyclodextrin based Nanosponge

\section{CLASSIFICATION OF NANOSPONGES:}

Nanosponges are encapsulating type of nanoparticles which encapsules the drug molecules within its core. By method of associating with drugs, the nanoparticles can be classified into the following:-

\section{Encapsulating nanoparticles}

This type is represented by nanosponges and nanocapsules. Nanosponges such as alginate nanosponge, which are sponge like nanoparticless containing many holes that carry the drug molecules in their aqueous core.

E.g. Nanosponges such as alginate nanosponge, which are sponge like nanoparticles containing many holes that carry the drug molecules. Nanocapsules such as poly (iso-butyl-cyanoacrylate) (IBCA) are also encapsulating nanoparticles.

\section{Complexing nanoparticles}

This type of nanoparticles attracts the molecules by electrostatic charges.

\section{Conjugating nanoparticles}

This type of nanoparticles links to drugs through covalent bonds. As compared to the other nanoparticles, they are insoluble both in water and organic solvents, porous, non-toxic and stable at high temperature up to $300{ }^{\circ} \mathrm{C}$. They are able to capture, transport and selectively release a huge variety of substances because of their 3D structure containing cavities of nanomeric size.

\subsubsection{Types of Nanosponges:}

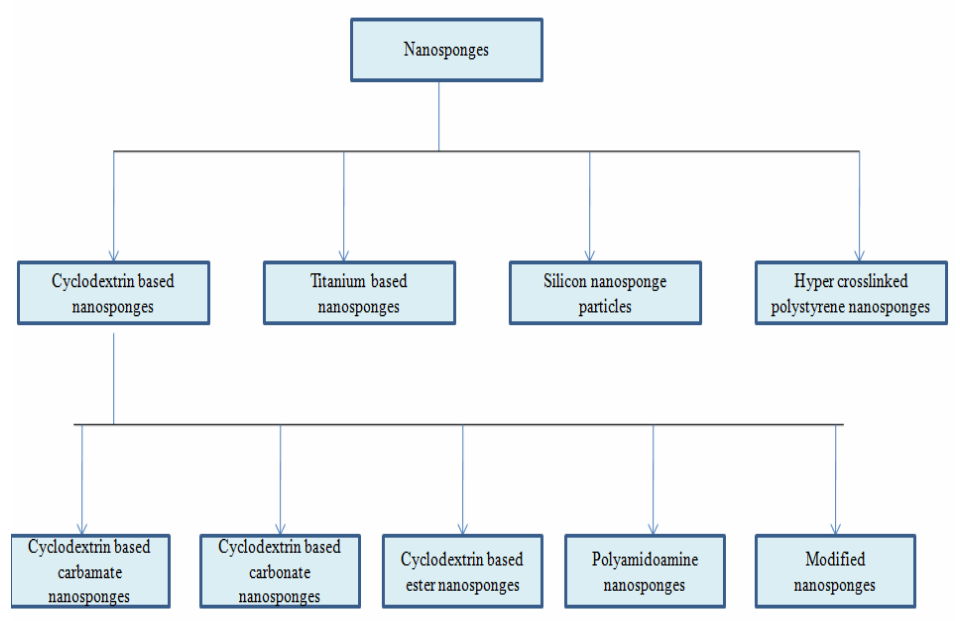

Fig 3: Types of Nanosponge

Significance of Nanosponges:

$>$ They are biodegradable, non - irritating, non-toxic. 
Indo Global Journal of Pharmaceutical Sciences, 2016; 6(2): 59-64

$>$ Size of the nanosponges can be varied by modifying/changing the ratio of cross- linker to polymer.

$>$ These are capable of carrying both lipophilic as well as hydrophilic drugs.

$>$ Possible predictable release. Provides extended release up to $12 \mathrm{hrs}$.

$>$ Protects the active ingredient from degradation.

$>$ Improved stability, elegance and formulation flexibility, the drug release profiles can be varied from fast, medium to slow release.

$>$ These are used as nano carriers for biomedical applications.

$>$ They can be used to mask unpleasant flavors and to change liquid substances into soilds.

$>$ These could be used to increase aqueous solubility of poorly water solubility.

$>$ They are stable at the $\mathrm{pH}$ range of 1-11.

\section{Formation of Nanosponges:}

Nanosponges possess a three-dimensional network or scaffold phenomenon. These are prepared by reacting polyesters (Cyclodextrin) with appropriate crosslinking agents, a novel nanostructured material can be obtained, known as nanosponges.

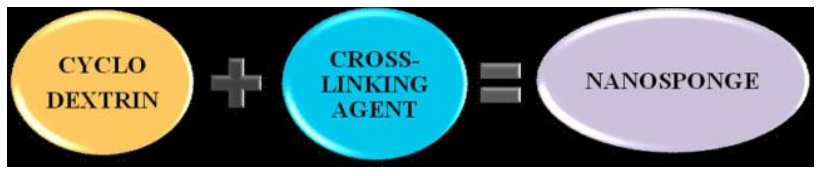

\section{Methods used for the preparation of Nanosponges:}

Following methods are used to prepare Nanosponges.

$>$ Melt Method

$>$ Solvent diffusion methods

- Emulsion solvent diffusion method

- Quasi-emulsion solvent diffusion.

$>$ Solvent method

$>$ Ultra Sound Assisted Method.

\section{Melt Method}

Nanosponges are prepared by reacting cyclodextrin with a cross- linker like: dimethyl carbonate, diphenyl carbonate, diisocyanates, diaryl carbonates, carbonyl diimidazoles, carboxylic acid anhydrides and 2, 2-bis (acrylamido) acetic acid.All the ingredients are finely homogeniszed, placed in a $250 \mathrm{ml}$ flask and heated at $100^{\circ} \mathrm{C}$. The reaction was carried out for about 5 hrs by using magnetic stirrer.The mixture was allowed to cool and broken down the product.
The obtained product was washed with suitable solvent to remove extra unreacted excipients and by products. The porosity, pore sizes and surface charge density of nanosponges can be controlled to attach different molecules.

\section{Solvent Diffusion Method}

a) Emulsion solvent diffusion method

In this method the two phases are prepared, Organic Phase and Aqueous Phase.

Aqueous phase consists of polyvinyl alcohol. Organic phase include drug and polymer.

After dissolving drug and polymer to suitable organic solvent (e.g.-dichloromethane), this phase is added slowly to the aqueous phase and then the mixture is stirred at $1000 \mathrm{rpm}$ for 2 hours. Nanosponges are collected by filtration washed. Dried such product in air at room temp or in an oven $40^{\circ} \mathrm{C}$ for 24 hours

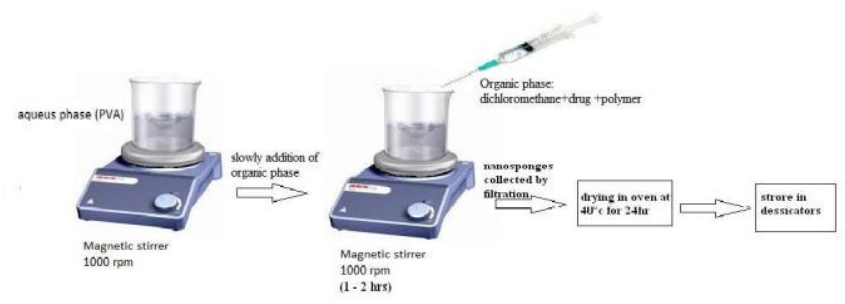

Fig 4. Pictorial representation of the preparation of nanosponges by solvent evaporation method.

\section{b) Quasi-emulsion solvent diffusion}

The nanosponges can also be prepared by quasi-emulsion solvent diffusion method using the different polymer amounts. To prepare the inner phase, eudragit RS100 was dissolved in suitable solvent. Then, drug can be added to the solution and dissolved under ultrasonication at 350c. The inner phase was poured into the PVA solution in water (outer phase) and allowed for stirring for $1 \mathrm{hr}$, then the mixture is filtered to separate the nanosponges. The nanosponges are dried in an air-heated oven at $40^{\circ} \mathrm{c}$ for $12 \mathrm{hrs}$.

\section{3) Solvent Method}

The solvent mixed with the suitable polymer, particularly in a polar aprotic solvent.e.g. Dimethylsulfoxide (DMSO), Dimethylformamide (DMF). This mixture added to the excess quantity of cross linkers e.g. cyclodextrine, Carbonyl compound (like: Dimethylcarbonate and Carbonyl diimidazole) preferably in following cross linker/polymer molar ratio of 4:16. Carried out the reaction at about $100^{\circ} \mathrm{c}$ temperature ranges with reflux temperature of the solvent, followed for the time ranges from $1 \mathrm{hr}$ to $48 \mathrm{hrs}$. 
Indo Global Journal of Pharmaceutical Sciences, 2016; 6(2): 59-64

When reaction was completed allow the solution to cool at room temperature.

After cooling added the product to the large excess of distilled water and recover the product through filtration under vaccum.

Then subsequently purify the product through Soxhlet Extraction by ethanol. Dry the product under vaccum and grind within a mechanical mill to obtain homogeneous powder.

\section{4) Ultrasound Assisted Synthesis}

In this method nanosponges can be obtained by reacting polymers with cross-linkers in the absence of solvent and under sonication. The nanosponges were produced by this method will be spherical and uniform in size.Then the polymer and the cross-linker in a particular molar ratio, mixed in a flask. Above flask placed in an ultrasound bath filled with water and heat upto $90^{\circ} \mathrm{C}$ and Sonicate this mixture for 5 hours. Then allow this mixture to cool and the product was broken roughly.

The product was cleaned with water to remove the non reacted polymer and purified by Soxhlet Extraction with Solvent (ethanol). After that, obtained product was dried under vacuum and stored at $25^{\circ} \mathrm{C}$ until further use.

\section{CHEMICALS USED FOR THE SYNTHESIS OF NANOSPONGES}

\section{a. Polymers}

- Hyper cross linked Polystyrenes,

- Cyclodextrines and its derivatives like: Methyl $\beta$-Cyclodextrin, Alkyloxycarbonyl Cyclodextrins, 2-Hydroxy Propyl $\beta$-Cyclodextrins

- Copolymers like Poly(valerolactone-allylvalerolactone) \& Poly(valerolactone-allylvalerolactoneoxepanedione) and Ethyl Cellulose \& Poly Vinyl Acryline.

\section{b. Crosslinkers}

- Diphenyl Carbonate,

- Diarylcarbonates,

- Diisocyanates,

- Pyromellitic anhydride,

- Carbonyldiimidazoles,

- Epichloridrine,

- Carboxylic acid dianhydrides,

- 2,2- bis(acrylamido) Acetic acid and

- Dichloromethane

\section{Drugs formulated as nanosponges}

- Itraconazole, Voriconazole, ketoconazole and meconazole as antifungal.

- $\beta$-Cyclodextrin Copolyvidonum

- Camptothecin $\beta$-Cyclodextrin Cancer.

- Dexamethazone $\beta$-Cyclodextrin Brain tumors.

- Econazole nitrate

- Ethyl Cellulose Polyvinyl alcohol

- Paclitaxel $\beta$-Cyclodextrin Cancer

- $\quad$ Resveratrol $\beta$-Cyclodextrin in cancer, cardiovascular diseases, inflammation, dermatitis etc.

- Inflammation Cardiovascular diseases Dermatitis Gonorrhea Fever Hyperlipidemia

- Tamoxifen $\beta$-Cyclodextrin Breast Cancer

- Temozolamide in brain tumour.

\section{EVALUATION OF NANOSPONGES}

\section{Solubility studies}

The phase solubility method is the used to study about inclusion complexation, which examines the effect of nanosponges on the solubility of drug. In this method, the drug was placed into an Erlenmeyer flask. This flask contains an aqueous solution of various percentages of nanosponges.. The solution thus obtained was analyzed to determine the concentration of drug by HPLC.

\section{Microscopy Studies}

For the study of microscopic aspects of drug, nanosponges and the product, scanning electron Microscopy (SEM) and Transmission Electron Microscopy (TEM) can be used.

\section{Determination of loading efficiency}

The measured quantity of loaded nanosponges complexes is dissolved in suitable solvent, sonicated to break the complex, diluted and after that analyzed by UV spectrophotometer, Loading efficiency can be estimated by following formula -

$$
\text { Loading Efficiency }=\frac{\text { Mctual drug control In }}{\text { Theoritical drug content }} \times 100
$$

\section{Infrared spectroscopy}

For estimating the interaction between nanosponges and drug molecules in the solid state, infra red spectroscopy is used. This technique is not suitable to find the inclusion complexes and also is less clarifying than other methods. 


\section{Determination of zeta potential}

Zeta potential is the measurement of surface charge. It can be measured by using additional electrode in the particle size equipment.

\section{X-ray diffractometry}

For detecting inclusion complexation in the solid state, powder $\mathrm{x}$-ray diffractometry is used. When the drug molecule is in liquid form, the diffraction pattern of a newly formed substance differs from uncomplexed nanosponge. This difference of diffraction pattern shows the complex formation. When the drug is in the solid form, a comparison can be made between the diffractogram of the assumed complex and mechanical mixture of the drug and polymer.

\section{Single crystal $x$-ray structure}

For determining the detailed inclusion structure and mode of interaction, single crystal x-ray structure analysis is used.

\section{Determination of Particle size}

For optimization process, the particle size of nanosponges is an important factor. Particle size can be estimated by laser light diffractometry or zeta sizer. Particles size greater than 30 $\mathrm{m}$ can appear gritty feeling while particles sizes range from 10 - $25 \mathrm{~m}$ are preferred for final topical formulation.

\section{In vitro release studies}

The releasing of drug from the optimized Nanosponge formulation can be studied by using multi compartment rotating cell with dialysis membrane. The donor phase as well as the receptor phase have drug loaded nanosponge complex in distilled water. The receptor phase is withdrawn after limited period of time, diluted with distilled water and analyzed by UV spectrophotometer.

\section{Photo degradation studies}

The photo degradation of drug loaded nanosponge is done under UV lamp. All the samples are put at distance of $10 \mathrm{~cm}$ from the lamp for 1 hour stirring under dark, and then the samples are analyzed by HPLC.

\section{Thermoanalytical Method}

These methods determine whether the changes occur in drug substance before the thermal degradation of the nanosponges includes (DTA and DSC) and the compatibility of drug with adjuvants can be determined by Thin Layer Chromatography (TLC) and Fourier Transform Infra-red Spectroscopy (FT-IR). Crystalline characteristics can be studied by powder X-ray diffraction (XRD) and Differential Scanning Colorimetric analysis. ical Sciences, 2016; 6(2): 59-64 CONCLUSION

The nanosponge is an effective targeted drug delivery system for poorly water soluble and variety of drugs such as: hydrophilic and lipophilic. Nanosponge technology involve encapsulation of medicament in a polymeric material in an innovative way and thus provide controlled site specific drug release, increased formulation efficacy, improved stability, drug dosing and patient compliance. Thus Nanosponge technology is the most promising leading trends in the area of pharmaceutical sciences for drug administration by oral, topical and parenteral route.

\section{REFERENCES}

1. Mark AM, Przemyslaw R, Greg C, Greg S, Akram S, Jonathan F et al. In vivo human time exposure study of orally dosed commercial silver nanoparticles. Nanomedicine: Nanotechnology, Biology, and Medicine. 2014, Vol.10, pp. 1-9.

2. Vyas SP, Khar RK. Novel carrier systems: Targeted and controlled drug delivery, 2002, 1st ed, New Delhi: CBS publishers \& distributors, pp. 332-413

3. Weisheng L, Yue wern H, Xiao DZ, Yinfa M. In vitro toxicity of silica nanoparticles in human lung cancer cells. Toxicol Appl Pharm., 2006, Vol. 217, pp. 252-259.

4. Subramanian S, Singireddy A, Krishnamoorthy K, Rajappan M. Nanosponges: a novel class of drug delivery system-review. J Pharm Pharm Sci., 2012, Vol. 15, Issue. 1, pp. 103-111.

5. Guo L, Gao G, Liu X, Liu F. Preparation and characterization of TiO2 nanosponge. Mater Chem Phys. 2008, Vol. 111,pp. 322325.

6. Trotta F, Zanetti M, Cavalli R. Cyclodextrin-based nanosponges as drug carriers. Beilstein J Org Chem, 2012, Vol. 8, pp. 20912099.

7. Swaminathan S, Vavia PR, Trotta F, Cavalli R, Tumbiolo S, Bertinetti L, et al. Structural evidence of differential forms of nanosponges of beta-cyclodextrin and its effect on solubilization of a model drug. J Incl Phenom Macrocycl Chem, 2012,Vol. 76, pp. 201-211.

8. Patel EK, Oswal RJ. Nanosponge and microsponges: a novel drug delivery system. Int J Res Pharm Chem, 2012.Vol.2, Issue.2, pp.237-244.

9. Vrushali Tamkhane, P.H. Sharma. Nanosponge-ANovel Drug Delivery System. Int. Curr. Pharm. J. Res. 2014, Vol. 4, Issue. 3, pp. 1186-1193.

10. Richhariya Neha, Dr. Prajapati S.K., Dr. Sharma U.K. Nanosponges: an innovative drug delivery system. World Journal of Pharmaceutical Research. 2015, Vol. 4, Issue. 7, pp. 17471759.

11. Carter SJ: Disperse system In: Cooper and Gunn's Tutorial Pharmacy. 6th ed. New Delhi: CBS Publishers and Distributors, 2000, pp. 68-72.

12. Kaur Loveleen Preet, Guleri Tarun Kumar, Topical Gel: A Recent Approach for Novel Drug delivery. Asian Journal of Biomedical and Pharmaceutical Sciences, 2013. Volume. 3, Issue 17, pp. 1-2.

13. Zatz, J.L. and Kushla, G.P. Gels. Lieberman, H.A., Rieger, M.M and Banker, G.S. Pharmaceutical dosage form: Disperse system, $2^{\text {nd }}$ edition, New York: Marcel Dekker. 2005, pp.399-421. 
Indo Global Journal of Pharmaceutical Sciences, 2016; 6(2): 59-64

14. S. Roychowdhury, D. H. Singh, R. Gupta, D. Manish, A Review on Pharmaceutical gel, Int. J. Pharma.Res. Bio., 2012, Vol. 1, Issue. 5, pp. 21-36.

15. Shah VP: Transdermal drug delivery system regulatory issues. In: Guy R.H. and had graft J. (eds.), Transdermal drug delivery. Marcel Dekker, New York, 2003, pp. 361-367.

16. Wilson and Ross, Waugh A. \& Grant A., Anatomy and Physiology in Health and Illness,Churchill Livingstone Elsevier Limited, $10^{\text {th }}$ edition, 2006. pp. 358.

17. Trotta F, Cavalli R. Characterization and application of new hyper-crosslinked cyclodextrins. Compos Interfaces., 2009, Vol. 16, pp. 39-48.

18. Shinde G, Rajesh KS, Bhatt D, Bangale G, Umalkar D, Virag G. Current status of colloidal system (nano range). Int Journal Drug Formul Res., 2011, Vol.2, Issue.6, pp. 39-54.

19. Guo L, Gao G, Liu X, Liu F. Preparation and characterization of TiO2 nanosponge. Mater Chem Phys. 2008, Vol. 111, pp. 322325.

20. Cavali R, Trotta F, Tumiatti V. Cyclodextrin based nanosponges for drug delivery. J Incl Phenom Macrocyl chem., 2006, Vol. 56, pp. 209-213.

21. Dakankov V, Llyin M, Tsyurupa M, Timofeeva G, Dubronina L. From a dissolved polystyrenecoil to intramolecularly hypercrosslinked nanosponges. Macromolecules. 1998, Vol. 29, pp. 8398-8403.

22. Swaminathan S, Pastero L, Serpe L, Trotta F, Vavia P, Aquilano $\mathrm{D}$ et al. Cyclodextrin based nanosponges encapsulating camptothecin: Physicochemical characterization, stability and cytotoxicity. Eur J Pharm Biopharm., 2010, Vol. 74, pp. 193-201.

23. Mamba B, Krause R, Malefetse T, Sithhole S. Cyclodextrin nanosponges in the removal of organic matter to produce water for power generation. Water SA. 2008, Vol. 34, pp. 657-660.

24. Trotta F, Cavalli R, Swaminathan S, Sarzanini C, Vavia P. Novel functionalized nanosponges synthesis, characterization. Safety assessment, cytotoxicity testing and interaction studies. Proceedings of the 14th International Cyclodextrin Symposium., Kyoto 2008, pp. 338-342.

25. Neha Richhariya, Dr. Sunil Kumar Prajapati, Dr. Upendra Kumar Sharma, 2015, Nanosponges: an innovative drug delivery system, World Journal of Pharmaceutical Research, Vol. 4, Issue.7, pp.1751-1753.

26. Bolmol U. B., Manvi F. V., Kotha Rajkumar, Palla S. S., Paladugu A, Reddy K R, Recent advances in nanosponge as drug delivery system, 2013,Vol.. 6, pp. 3.

Indo Global Journal of Pharmaceutical Sciences( ISSN 22491023 ; CODEN-IGJPAI; NLM ID: 101610675) indexed and abstracted in EMBASE(Elsevier), SCIRUS(Elsevier),CABI, CAB Abstracts, Chemical Abstract Services(CAS), American Chemical Society(ACS), Index Copernicus, EBSCO, DOAJ, Google Scholar and many more. For further details, visit 